\title{
COMPARISON OF FINISHING AND POLISHING SYSTEMS FOR RESIDUAL RESIN REMOVAL AFTER DEBONDING
}

\author{
Çaöry ULUSOY ${ }^{1}$
}

1- DDS, PhD, Research Assistant, Department of Orthodontics, Gazi University Faculty of Dentistry.

Corresponding address: 82. sokak Emek - 06510 - Ankara - TURKEY - Phone: +90.312.2034289 - e-mail: culusoy77@yahoo.com

Received: August 09, 2008 - Modification: November 07, 2008 - Accepted: November 16, 2008

\begin{abstract}
$O$

bjective: The aim of this study was to evaluate (1) the effectiveness of one-step polishers on the surface morphology of enamel using scanning electron microscope (SEM) and compare their effects with conventional systems for residual adhesive removal; and (2) the time spent to remove resin remnants. Material and Methods: Metal brackets were bonded to the buccal surface of 80 freshly extracted human premolar teeth and received the same resin-removal methods to evaluate the time spent to remove resin remnants $(\mathrm{n}=10)$. The brackets were debonded and residual adhesive was removed using different systems. Fourty-five premolars, including the control group with intact enamel $(n=5)$, were examined by SEM. Results: 30-blade tungsten carbide burs were the least timeconsuming procedure. The best system in SEM study was the PoGo micro-polishers followed by Super-Snap Rainbow system. Conclusions: The effect of polishing systems on residual resin removal was dependent on the characteristics of the instruments in each system.
\end{abstract}

Key words: Scanning electron microscopy. Orthodontic bracket debonding. Dental polishing

\section{INTRODUCTION}

Direct bonding of brackets onto the teeth revolutionized the orthodontic practice. The acid-etch technique creates a strong bond between the direct bonding materials and enamel, but debonding of the brackets and improper finishing and polishing at the end of treatment have the potential to cause iatrogenic enamel damage ${ }^{4}$, excessive plaque accumulation, gingival irritation and increased surface staining ${ }^{8,20}$. After removal of bonded orthodontic appliances, certain procedures can be considered with the goal of achieving an ideal enamel surface layer in structure and appearance. Previous studies used different methods for cleaning the residual resin after removal of orthodontic attachments ${ }^{2,5,12,14}$. Some practitioners prefer conventional diamond burs for the removal of composite remnants, but regardless of how careful the resin is cut, these burs may scratch the enamel due to their shape and sharpness. Retief and Denys ${ }^{13}$ reported that conventional diamond finishing burs should not be used for residual resin removal after debracketing because they cause deep gouges on the enamel surface. Radlanski ${ }^{12}$ showed that conventional carbide burs may scratch the enamel due to the shape and sharpness of their blades.

Currently, many finishing and polishing devices have been proposed ${ }^{2,3,5-7,12-15,17,18,22,25}$. Use of multi-step systems including fine and superfine tungsten carbide burs (TCB) and aluminum oxide coated abrasive disks are the first considerations. On the other hand, using multi-step systems seems to be time-consuming and each of these devices leaves the surface of dental enamel with varying degrees of surface roughness ${ }^{2,5}$. One-step polishing systems, including diamond impregnated rubber cups, points and silicon carbide brushes were introduced for finishing and polishing resin composites to a smooth and glossy appearance , $^{3-11,19-21}$. With the ultimate goal of achieving a smooth surface in fewer steps, one-step polishing systems seem to be promising to the clinician for the removal of residual resin after debracketing.

Information about the effect of different systems that would return the enamel surface as closely as possible to its original state after removal of orthodontic brackets seems to be limited in the literature. The search for the ideal method that returns the enamel surface as closely as possible to its original state is still ongoing. To date, no study has been performed evaluating the effect of one-step micro-polishers on the residual resin removal after debonding of orthodontic brackets.

The purpose of this in vitro study was to evaluate (1) the effectiveness of one-step diamond and silicone carbide impregnated polishers on the surface morphology of enamel using scanning electron microscope (SEM) and compare their effects with conventional systems for residual adhesive removal and (2) the time spent to remove resin remnants. 


\section{MATERIAL AND METHODS}

In this in vitro study, 85 freshly extracted human premolar teeth were collected and stored at room temperature in distilled water containing $0.1 \%$ thymol crystals to inhibit bacterial growth until their use in two weeks. The teeth were cleaned and polished with fluoride-free pumice slurry and examined under a stereo microscope (Discovery V8 Stereo, Carl Zeiss MicroImaging GmbH, Göttingen, Germany) to ensure the absence of caries and cracks on the labial surface. The crowns were washed and dried for $15 \mathrm{~s}$. Except for the control group for the scanning electron microscope study $(n=5)$, the buccal enamel of 80 teeth was etched with $37 \%$ orthophosphoric acid (3M ESPE, St. Paul, Minnesota, USA) for $30 \mathrm{~s}$, rinsed with water for 15 seconds and air dried with oil-free compressed air. Each premolar metal orthodontic bracket with 0.018-inch slot (Victory Series, 3M Unitek $\mathrm{GmbH}$, Seefeld, Germany) was centered on the crown of the tooth mesiodistally and along the long axis of the tooth and bonded using Transbond XT primer and adhesive paste (3M/Unitek, Monrovia, California, USA) according to manufacturer's recommendation with a halogen curing unit (Hilux Ultra Plus, Benlioðlu Dental, Ankara, Turkey). Before each procedure, the light intensity of the unit was checked with a curing radiometer (Demetron, Kerr, Danburry, CT, USA) as $700 \mathrm{~mW} / \mathrm{cm}^{2}$. The excess bonding resin around the bracket was removed using a sharp scaler. After light irradiation from mesial and distal bracket edges for $10 \mathrm{~s}$, the specimens were kept in distilled water at $37^{\circ} \mathrm{C}$ for $24 \mathrm{~h}$ to allow polymerization of resin. The brackets were removed using a plier (Dentaurum, Pforzheim, Germany) and residual adhesive was removed by using different systems. The teeth receiving the same resin-removal methods were randomly divided into four groups to evaluate the time spent to remove resin remnants $(\mathrm{n}=20)$.

The enamel treatments of the specimens for the time spent to remove residual resin were as follows:

Group 1- Burs $(\mathrm{n}=20)$

a- 12-fluted tapered TCB (Hager\& Meisinger GmbH,
Neuss, Germany) with a brush stroke by high-speed hand piece with water cooling $(\mathrm{n}=10)$

b- 30-fluted tapered TCB (Axis Dental, Irving, TX, USA) with a brush stroke by high-speed hand piece with water cooling $(\mathrm{n}=10)$

Group 2- Discs $(\mathrm{n}=20)$

a- coarse, medium, fine, and superfine aluminum-oxide abrasive Sof-Lex discs (3M-ESPE Dental Products, St. Paul, MN, USA) by low-speed hand piece with air cooling $(\mathrm{n}=$ 10)

b- coarse, medium, fine, and superfine aluminum-oxide abrasive Super-Snap discs (Shofu Dental Corp, Kyoto Japan) by low-speed hand piece with air cooling $(n=10)$

Group 3- Brushes $(\mathrm{n}=20)$

a- diamond coated PoGo micro-polisher point (Dentsply Caulk, Milford DE, USA) by low-speed hand piece with air cooling $(\mathrm{n}=10)$

b- silicone carbide impregnated OptiShine brush (KerrHawe SA, Bioggio, Switzerland) by low-speed hand piece with air cooling $(\mathrm{n}=10)$

Group 4- Combination of burs and brushes $(n=20)$

a- a sequence of 30-fluted tapered TCB and diamond coated PoGo micropolisher point $(\mathrm{n}=10)$

b- a sequence of 30-fluted tapered TCB and silicone carbide impregnated OptiShine brush $(\mathrm{n}=10)$

The removal of the composite was considered complete when the tooth surface seemed smooth and free of composite to the naked eye under the light of an operative lamp ${ }^{14}$. Each $\mathrm{TCB}$, disc, diamond and silicone impregnated polisher were discarded after use. After the use of each disc, the tooth surfaces were rinsed and dried before proceeding to the next grit. A dry field was maintained for the use of each grit disc.

The results of the time test were subjected to one-way analysis of variance (ANOVA). The Bartlett test revealed that there was no homogenity among the groups $(P<0.01)$. Differences between the data were evaluated by a Duncan test (Table 1).

For SEM evaluation, fourty-five samples were examined in five groups by scanning electron microscope. The first

TABLE 1- Time needed for cleaning the residual adhesive after debonding of brackets (seconds)

\begin{tabular}{|c|c|c|c|c|c|c|c|}
\hline Group & $\mathbf{n}$ & Mean & & SE of Mean & SD & Minimum & Maximum \\
\hline 12-fluted tungsten carbide bur & 10 & 8.414 & $\mathrm{~F}$ & 0.188 & 0.594 & 7.560 & 9.390 \\
\hline 30-fluted tungsten carbide bur & 10 & 5.256 & G & 0.053 & 0.167 & 5.010 & 5.510 \\
\hline Sof-Lex multi-step disc & 10 & 31.006 & C & 0.419 & 1.325 & 29.070 & 33.020 \\
\hline SuperSnap multi-step disc & 10 & 30.556 & C & 0.390 & 1.232 & 28.770 & 32.200 \\
\hline Pogo one-step micropolisher & 10 & 38.288 & $A$ & 0.480 & 1.517 & 36.480 & 41.200 \\
\hline Optishine one-step brush & 10 & 33.115 & B & 0.495 & 1.564 & 30.900 & 35.710 \\
\hline $\begin{array}{l}\text { 30-fluted tungsten carbide bur } \\
\text { +Pogo one-step micropolisher }\end{array}$ & 10 & 14.838 & $E$ & 0.153 & 0.483 & 14.070 & 15.530 \\
\hline $\begin{array}{l}\text { 30-fluted tungsten carbide bur } \\
\text { +Optishine one-step brush }\end{array}$ & 10 & 15.628 & $\mathrm{D}$ & 0.314 & 0.994 & 14.320 & 16.880 \\
\hline
\end{tabular}

$\mathrm{n}$ : number of specimens; SE: Standard error; SD: Standard deviation.

Different letters indicate statistically significant difference between the mean values of the groups. 
four groups which used the same resin-removal methods as mentioned above, were randomly divided into eight subgroups $(\mathrm{n}=5)$. The fifth group had no treatment and the intact enamel surfaces acted as control $(n=5)$. The specimens were then placed on a rotating table in a high vacuum evaporator and coated with $250 \mathrm{~A}^{\circ}$ of gold. The specimens were examined with SEM (JSM 6400, JEOL Ltd, Fukuoka, Japan) with a magnification of $x 500$ and $x 2,500$ at an accelerating voltage of $20 \mathrm{kV}$.

\section{RESULTS}

The time spent to remove resin remnants in all groups was evaluated by one-way ANOVA and Duncan test. Except for the Super Snap and Sof-Lex disc groups.,there were statistically significant differences among the experimental groups $(P<0.05)$ for the time spent in removing the residual resin (Table 1). The maximum time required to clean the surfaces was found when one-step diamond coated Po-Go micro-polisher was used without any pretreatment (Group 3a). Pretreatment with a 30 -fluted TCB led to clean the residual remnants from the enamel surface with PoGo micro-polishers in a shorter time period. The minimum time was found when 30-fluted TCB was used to clean the residual resin.

SEM analysis of the enamel surfaces after the use of onestep and multi-step systems are shown in Figures 1- 8. Figure 9 shows SEM analysis of the intact enamel.

In the bur groups, 12-fluted TCB resulted in an irregular enamel surface in SEM evaluation, showing horizontal scars with a consistent pattern (Figure 1). 30-fluted TCB also resulted in less scarring on the enamel surface. On the other hand, both burs left remnants on the enamel surfaces (Figures 1 and 2).

In the disc groups, residual resin removal with Sof-Lex aluminum oxide abrasive discs showed a decrease in surface irregularities but scratches were seen in every direction (Figure 3). Super Snap discs produced a smooth and homogeneous enamel surface with less amount of scratches on the enamel surface (Figure 4).

In the brush groups, the one-step diamond coated PoGo micro-polisher produced the best surface without any scratches, although a few remnants were observed on the surface (Figure 5). Silicone carbide impregnated one-step OptiShine brush caused severe roughness with islets of residual resin remnants on the enamel surface. Also, a lot of worn out bristles were observed on the enamel surface (Figure 6).This method was not found to be efficient for cleaning the enamel surfaces after debonding of brackets.

In the combination groups, PoGo micro-polishers used in combination with 30 -fluted tapered TCB resulted in relatively smooth surfaces, but this method was not efficient in removing the scratches on the enamel surface produced by the bur (Figure7). OptiShine brush applied after 30-fluted tapered TCB resulted in smoother surfaces than group 3-b specimens. On the other hand, the scars on the enamel surface produced by the bur could not be efficiently removed by this method (Figure 8).

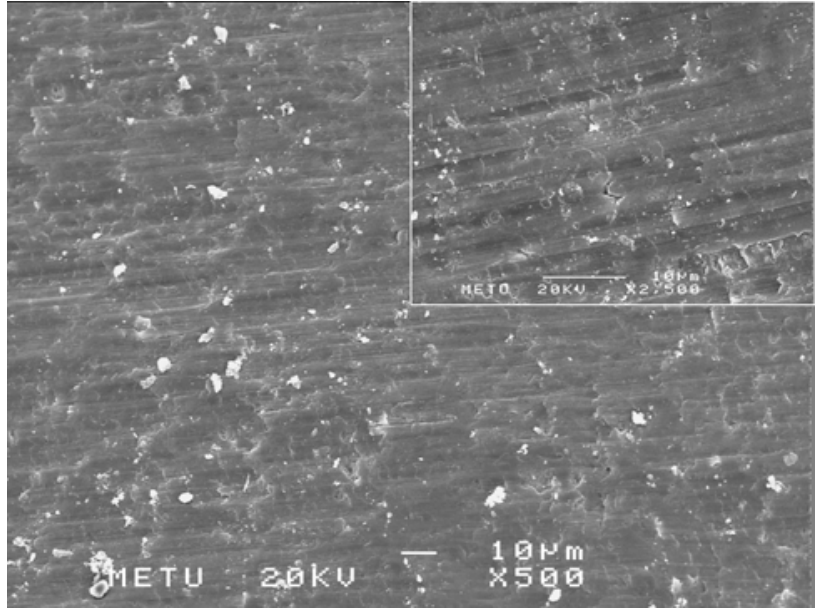

FIGURE 1- SEM analysis of the enamel surface after using 12-fluted tungsten carbide bur

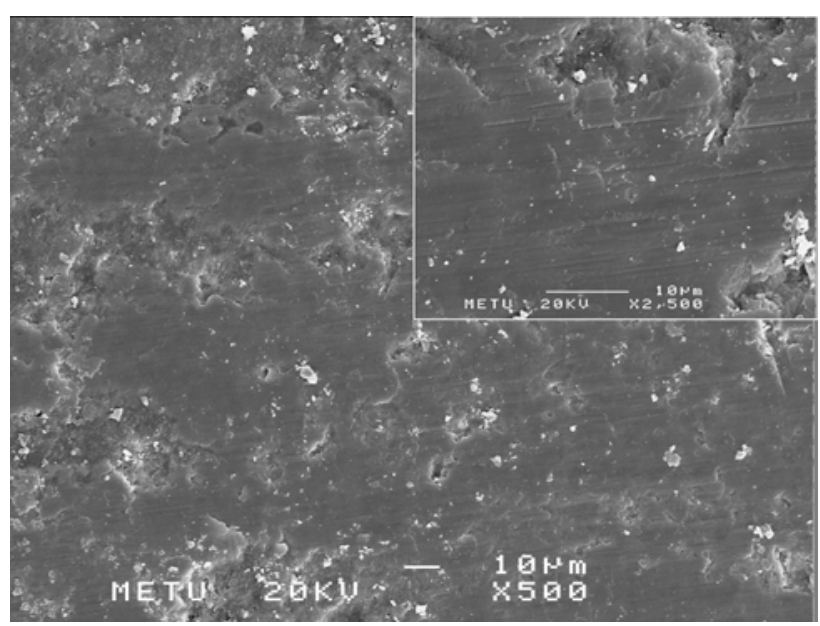

FIGURE 2- SEM analysis showing enamel surface after using 30 -fluted tungsten carbide bur for surface cleaning

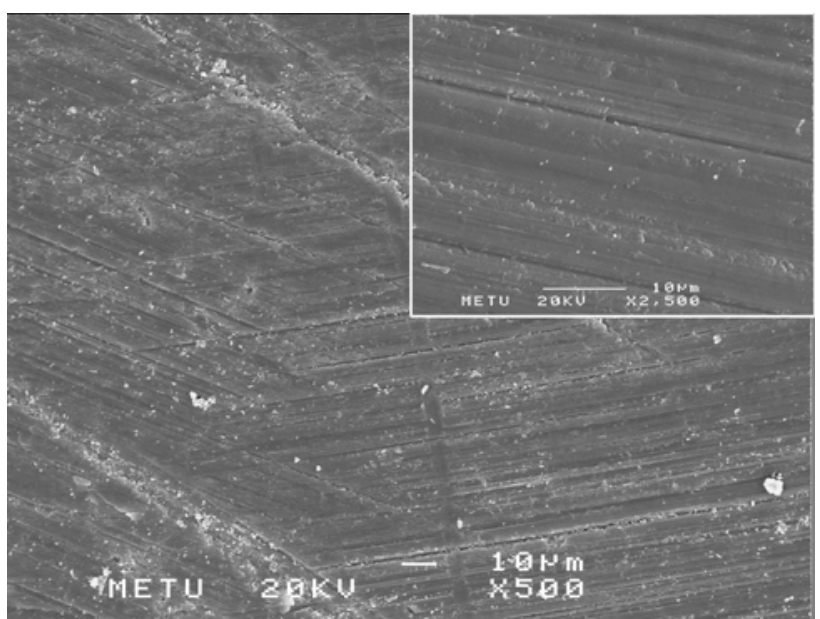

FIGURE 3- SEM analysis showing the enamel surface after residual resin removal with Sof-Lex aluminum oxide abrasive discs 


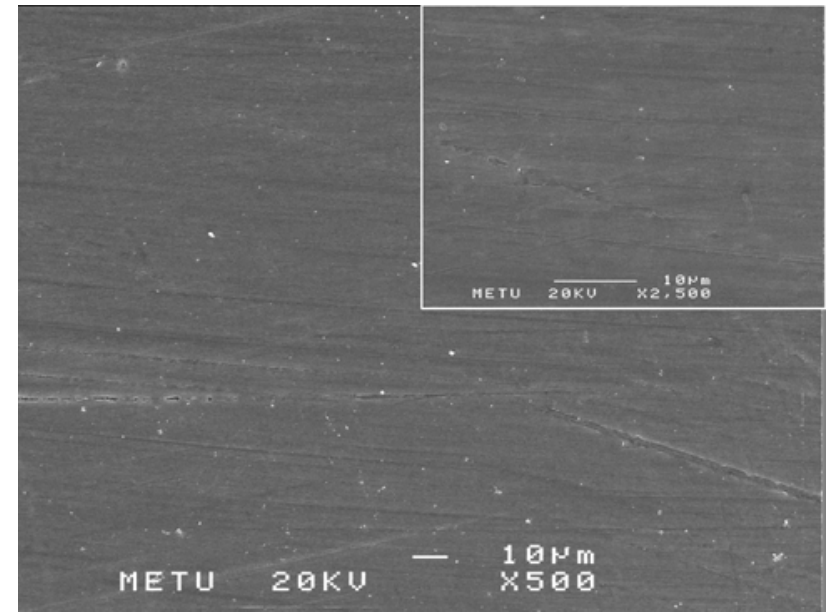

FIGURE 4- SEM analysis of the enamel surface after using Super-Snap Rainbow discs

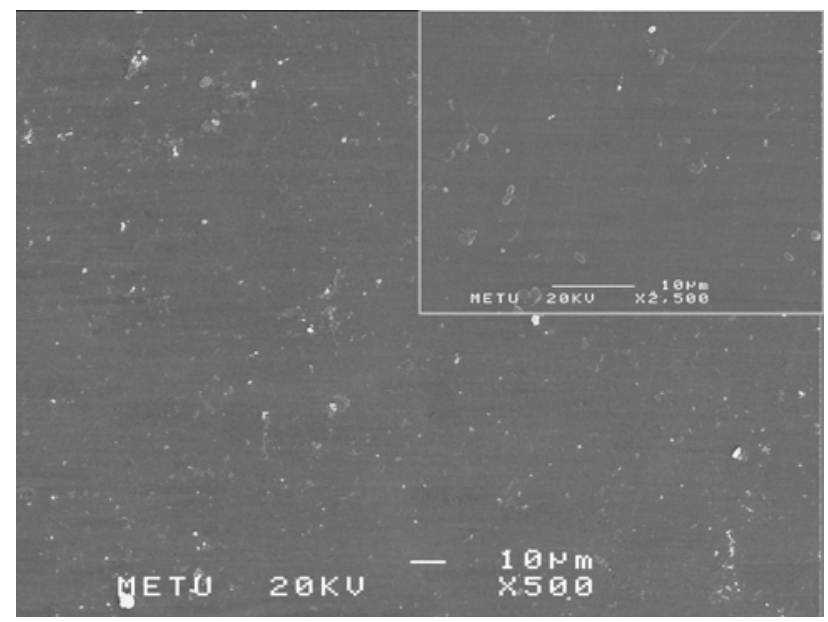

FIGURE 5- SEM analysis showing enamel surface after using diamond coated Po-Go micro-polisher point

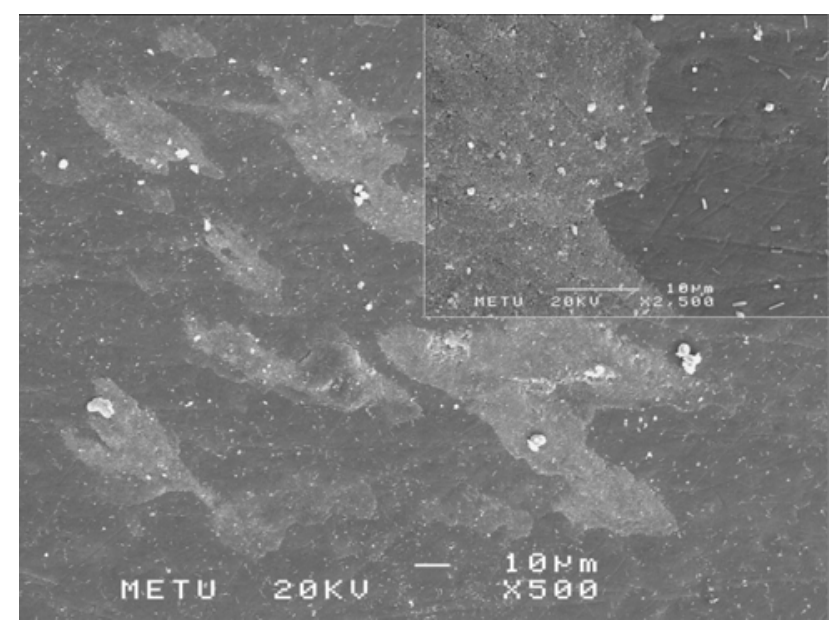

FIGURE 6- SEM analysis after residual remnants removal with silicone carbide impregnated one-step OptiShine brush

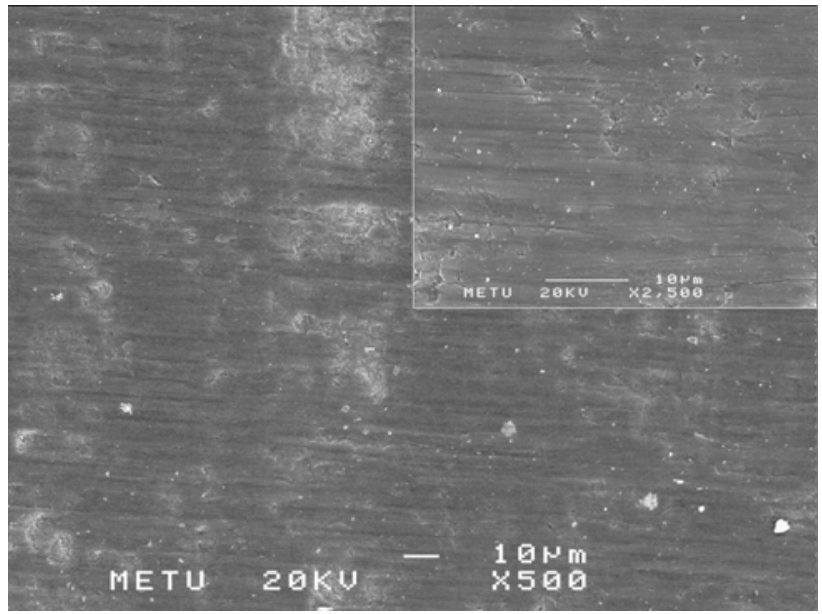

FIGURE 7- SEM analysis showing enamel surface after using 30-fluted tungsten carbide bur in combination with diamond coated one-step Po-Go micro-polisher point

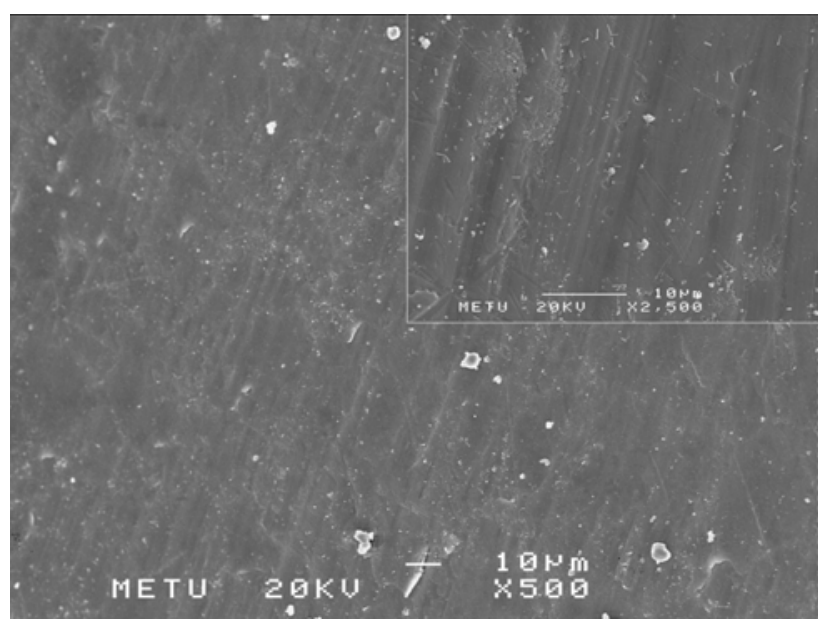

FIGURE 8- SEM analysis showing enamel surface after using 30-fluted tungsten carbide bur in combination with silicone carbide impregnated one-step OptiShine brush

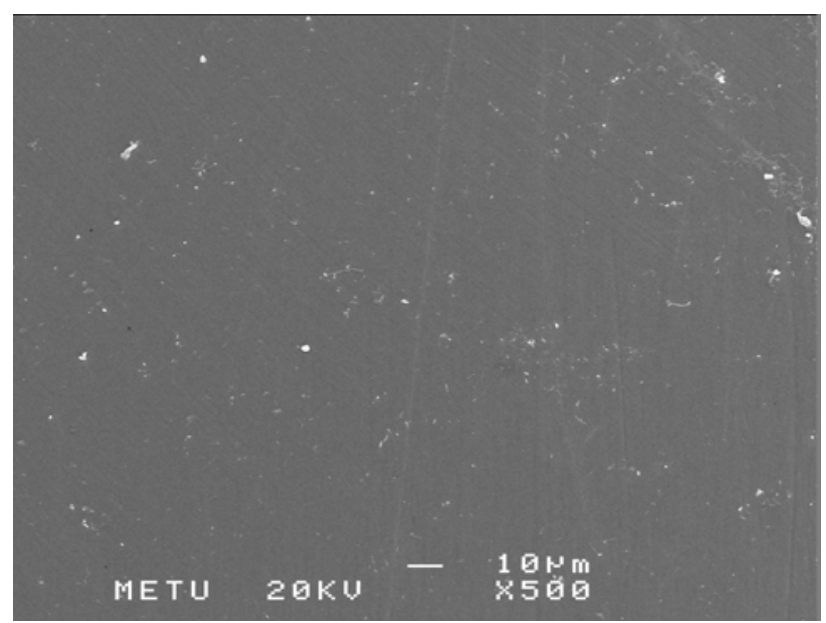

FIGURE 9- SEM analysis of the intact enamel 


\section{DISCUSSION}

Visualization of dental structures at the submicron and nanometer levels is essential for understanding the complex surface topography and failure modes of advanced technologies. Although SEM provides only a subjective and non-quantifiable information ${ }^{4}$, the effectiveness of various methods and instruments on the topography and morphology of the tooth surfaces are best examined under SEM. In this study SEM was used to examine the changes on enamel surface by using different rotary instruments for residual resin removal after debracketing and a time study was conducted to find out the time different methods require.

When the orthodontic treatment is finished, after debonding of brackets the ideal finish is desired for the tooth surface, without removing an excessive amount of tooth structure. Improper debonding results in cracks on enamel surface and enamel prism fractures. Esthetic problems, tooth sensitivity, increasing risk of caries and pulp necrosis may also be seen after improper debonding. In the literature were found few reports on comparing the enamel structure with different debracketing procedures ${ }^{1,14,25}$. Zarinnia, et al. ${ }^{25}$ showed that the bracket removing plier produced the most consistent separation at the bracket-adhesive interface. Except for the control group, the brackets in the present study were debonded by pliers in order to mimic the clinically debonded surfaces in vivo.

After the removal of bonded orthodontic appliances, certain procedures can be considered with the goal of achieving an ideal enamel surface layer in structure and appearance. After debonding of brackets, the mechanical removal of residual composite with rotary instruments, including various shapes of tungsten-carbide burs or SofLex discs, may damage the enamel ${ }^{2,7}$. The search for the best method has resulted in the introduction of new instruments and procedures, including carbon-dioxide ${ }^{15}$ and $\mathrm{Nd}$ :YAG laser application ${ }^{18}$ for cleaning the enamel surface after debonding of brackets. Air-powder abrasive systems are also suggested for removing residual adhesive ${ }^{18}$, but the need for rubber dam application and protective mask is the impractical aspect of this technique ${ }^{22}$. The introduction of novel methods has resulted in the development of new instruments, such as specially designed burs, discs and diamond or silicone coated polishers which are thought to be less aggressive ${ }^{12,23}$. The primary concern after debonding of brackets is to return the enamel surface as closely as possible to its original state 2 .

TCBs are available in various sizes, shapes and different grits. The frequently employed ones have $8-30$ flutes $^{2,6}$ and 12- and 30-flutes are safer to use on enamel. In this study, 12-fluted TCB was found to be effective in residual resin removal in accordance with the Rouleau, et al. ${ }^{14}$ study. SEM micrographs clearly demonstrate that the enamel scarring is inevitable when 12-fluted TCB is used with high speed hand piece with water cooling. On the other hand, it is a fast method to remove the residual resin. In accordance with Zarrinnia, et al. ${ }^{25}$, carbide burs at high speed were found to be efficient in residual resin removal, but when used alone failed to produce a satisfactory enamel surface in the present study, by SEM evaluation. Zachrisson and Artun ${ }^{24}$ recommended using tungsten carbide burs, but at low speeds. Campbell ${ }^{2}$ and Rouleau, et al. ${ }^{14}$ recommended using tungsten carbide burs with water cooling at high speed, whereas Retief and Denys ${ }^{13}$ suggested adequate air cooling to observe the resin remnants during the cleaning of enamel surface. In this study, water cooling was preferred to air cooling to avoid intrapulpal temperature rise.

Campbell ${ }^{2}$ reported that using 30 -fluted tungsten carbide bur appeared to be the most efficient method of removing highly filled resin after debonding and it produced lesser amount of scars. Our results are in accordance with Campbell ${ }^{2}$. Cleaning the residual adhesive on enamel surfaces after debonding with 30-fluted TCB was found to be the least time-consuming method in the present study.

Discs have the reputation of providing the highest polish and most discs are coated with aluminum oxide abrasives ${ }^{20}$. Sof-Lex and Super Snap multi-step systems consist of four (coarse, medium, fine, ultra fine) discs. Sequential use, starting with the coarse grit and gradually progressing to the superfine grit is necessary and this is time-consuming 5 . Discs were used with a low-speed handpiece at a speed of $10,000 \mathrm{rpm}$ in the present study because higher speeds than $15,000 \mathrm{rpm}$ may generate excess heat, dislocate the disk and cause injury. Residual resin removal with Sof-Lex discs resulted in a decrease in surface irregularities according to the burs, but left abrasive remnants on the enamel surface. This result was in accordance with other authors ${ }^{13,25}$. The clinically observed metal traces along with the increased number of scratches and gouging on SEM micrographs may be attributed to the fact that the mandrel used with Sof-Lex discs has metal centers. Super Snap discs are snapped on and secured onto the mandrel by an elastic shank mounted on the discs. As there are no metal centers in the Super Snap discs, no metal exposure was observed clinically and few scratches were observed in group 2-b specimens, in the SEM examination. The undesired scratches observed in SEM evaluation of enamel surfaces with these two multi-step systems are generally caused by the edges of the discs. SuperSnap system showed the second-best results and resulted in an apparently better surface finish than the other multi-step system, Sof-Lex.

Recently, diamond or silicone carbide coated polishers were introduced in order to reduce the chair time. The onestep PoGo Polishers (discs, cups and points) are single-use diamond-impregnated polishing devices and designed for use without water in the final polishing of composite resin restorations ${ }^{19,21,23}$. Yap, et al. ${ }^{23}$ and St Georges, et al. ${ }^{16}$ used PoGo micropolishers on composite surfaces without any pretreatment. The same method was applied in this study for removing the residual adhesive on enamel surfaces in the group 3-a specimens. Although there were few remnants left on the enamel surface, which may possibly be the remainders of fine diamond powder integrated in the polishing device, the one-step diamond coated PoGo micropolishers produced the best surface finish without any scratches in the present study. Turkun, et al. ${ }^{19}$ showed that 
PoGo micropolishers produced surface roughness comparable to that of Mylar strips for resin composites. The results of the present study is consistent with Turkun et al ${ }^{19}$ and indicates that PoGo micropolishers when used without any preatreatment are effective in removing the residual remnants and return the enamel as closely as possible to its original state. On the other hand, this method was found to be the most time-consuming. PoGo micro-polishers used in combination with 30 -fluted TCB cleaned the residual remnants from the enamel surfaces in a shorter time, but this method was not efficient in removing the scratches on the enamel surface produced by the bur in group 4-a specimens.

OptiShine is a concave shaped brush with silicon carbide polishing particles embedded in the bristles. The manufacturer recommends using the OptiShine brush without a polishing paste. The present study revealed that one-step OptiShine brush, when used without any pretreatment on the enamel surface should be the least preferable method in removing the residual resin after debonding of brackets. The brush left islets of residual resin composite remnants and worn-out bristles on the enamel surface. OptiShine brush applied after 30-fluted tapered TCB resulted in smoother surfaces than group 6 specimens. On the other hand, this method was inefficient to remove the scars on the enamel surface produced by the bur. Jung, et al. ${ }^{9}$ reported that the abrasive potential of OptiShine brush was relatively low and the surface irregularities of resin composites followed by finishing with $30 \mu \mathrm{m}$ and $20 \mu \mathrm{m}$ diamonds were not sufficiently removed. If the initial finishing was done with a $30 \mu \mathrm{m}$ diamond and a tungsten carbide bur, OptiShine brushes produced smoother surfaces. Our results are in accordance with those of Jung, et al. ${ }^{9}$.

As heat builds up with prolonged contact with rotary instruments ${ }^{21}$, Sof-Lex and SuperSnap discs, PoGo micropolisher and OptiShine brush were used with light intermittent strokes on the enamel surface in the present investigation.

The efficiency order of the tested residual resin removal systems in SEM was as follows: PoGo one-step micropolisher without any pretreatment $>$ Super Snap multistep polishing system $>$ PoGo micropolisher used in combination with 30-fluted tungsten carbide bur $>$ OptiShine brush used in combination with 30 -fluted tungsten carbide bur $>$ Sof-Lex multi-step polishing system $>30$-fluted tungsten carbide bur $>12$-fluted tungsten carbide bur $>$ OptiShine brush without any pretreatment.

The minimum time was recorded for the 30-fluted tungsten carbide bur method. Maximum time required to clean the surfaces was observed when one-step diamond coated Po-Go micro-polisher was applied without any pretreatment. Although the results of this study indicated that it is possible to obtain enamel surfaces nearly as smooth as the intact or untreated enamel with PoGo one-step micropolisher point, it will take a long time to remove the residual resin from the enamel surface in a full-bonded case situation.

We strongly agree with Koh, et al. ${ }^{11}$ who reported that surface roughness could be determined by the characteristics of the polishing instruments. Even the similar polishing instruments in the same groups, one-step or multi-step, resulted in different enamel surface finish. This difference was probably due to the different ingredients of one-step polishing devices, different particle sizes of the multi-step discs and metal centers of Sof-Lex discs. In this study, all finishing systems were found to be clinically acceptable in removing the residual resin after debonding, except for the OptiShine brush group without any pretreatment. The overall findings of this study reveal that scarring of enamel after debonding procedures is inevitable but can be reduced by choosing the right protocol. Further studies are required where finishing techniques can be tested in clinical settings.

\section{CONCLUSIONS}

The effect of one-step and multi-step polishing systems on residual resin removal from the enamel was dependent on the characteristics of the instrument in each system.

12- and 30-fluted TCB at high speed and water coolant proved to be fast and efficient in residual resin removal, but the resultant enamel surface with enamel scars needs to be finished by other polishing techniques.

Following the use of TCB and multi-step disc systems for residual resin removal, scratching of enamel is inevitable.

Super Snap discs were less aggressive than Sof-Lex discs in removing residual bonding resin and resulted in apparently better surface finish causing less damage to the enamel.

One-step PoGo micropolishers resulted in enamel surfaces nearly as smooth as the intact enamel, but found to be the most time consuming method.

The results of this research indicated that one-step polishing systems should be used after cleaning the resin remnants on enamel with TCB to avoid extending the chairtime.

\section{REFERENCES}

1- Brosh T, Kaufman A, Balabanovsky A, Vardimon A. In vivo debonding strength and enamel damage in two orthodontic debonding methods. J Biomech. 2005;38:1107-13.

2- Campbell PM. Enamel surfaces after orthodontic bracket debonding. Angle Orthod. 1995;65:103-10.

3- Costa J, Ferracane J, Paravina RD, Mazur RF, Roeder L.The effect of different polishing systems on surface roughness and gloss of various resin composites. J Esthet Restor Dent. 2007;19:214-24.

4- Eliades T, Gioka C, Eliades G, Makou M. Enamel surface roughness following debonding using two resin grinding methods. Eur J Orthod. 2004;26:333-8.

5- Eminkahyagil N, Arman A, Çetinsahin A, Karabulut E. Effect of resinremoval methods on enamel and shear bond strength of rebonded brackets. Angle Orthod. 2006;76:314-21.

6- Hong YH, Lew KKK. Quantitative and qualitative assessment of enamel surface following five composite removal methods after bracket debonding. Eur J Orthod. 1995;17:121-8. 
7-Howell S, Weekes WT. An electron microscopic evaluation of the enamel surface subsequent to various debonding procedures. Aust Dent J. $1990 ; 35: 245-52$

8- Jefferies SR. The art and science of abrasive finishing and polishing in restorative dentistry. Dent Clin North Am. 1998;42:613-27.

9- Jung M, Eichelberger K, Klimek J. Surface geometry of four nanofiller and one hybrid composite after one-step and multiple-step polishing. Oper Dent. 2007;32:347-55

10- Jung M. Surface roughness and cutting efficiency of composite finishing instruments. Oper Dent. 1997;22:98-104.

11- Koh R, Neiva G, Dennison J, Yaman P. Finishing Systems on the Final Surface Roughness of Composites. J Contemp Dent Pract. 2008;138 45 .

12- Radlanski RJ. A new carbide finishing bur for bracket debonding. J Orofac Orthop. 2001;62:296-304.

13- Retief DH, Denys FR. Finishing of enamel surfaces after debonding of orthodontic attachments. Angle Orthod. 1979;49:1-10.

14- Rouleau BD, Grayson WM, Cooley RO. Enamel surface evaluations after clinical treatment and removal of orthodontic brackets. Am J Orthod. $1982 ; 81: 423-6$

15- Smith SC, Walsh LJ, Taverne AA. Removal of orthodontic bonding resin residues by $\mathrm{CO}_{2}$ laser radiation: surface effects. J Clin Laser Med Surg. 1999;17:13-8.

16- St Georges AJ, Bolla M, Fortin D, Mueller-Bolla M, Thompson JY, Stamatiades PJ. Surface finish produced on three resin composites by new polishing systems. Oper Dent. 2005;30:593-7.

17- Sundfeld RH, Rahal V, Croll TP, De Alexandre RS, Briso AL. Ename microabrasion followed by dental bleaching for patients after orthodontic treatment — case reports. J Esthet Restor Dent. 2007;19:71-7.

18- Thomas BW, Hook CR, Draughn RA. Laser-aided degradation of composite resin. Angle Orthod. 1996;66:281-6.

19- Türkün LS, Türkün M. The effect of one-step polishing system on the surface roughness of three esthetic resin composite materials. Oper Dent. 2004;29:203-11.

20- Uçtaşli MB, Arisu HD, Omürlü H, Eligüzeloölu E, Ozcan S, Ergun G. The effect of different finishing and polishing systems on the surface roughness of different composite restorative materials. J Contemp Dent Pract. 2007;8:89-96.

21- Uysal T, Eldeniz AU, Usumez S, Usumez A.Thermal changes in the pulp chamber during different adhesive clean-up procedures. Angle Orthod. 2005;75:220-5.

22- Wright GZ, Hatibovic-Kofman S, Millenaar DW, Braverman I. The safety and efficacy of treatment with air abrasion technology. Int J Paediatr Dent. 1999;9:133-40

23- Yap AU, Yap SH, Teo CK, Ng JJ. Finishing/Polishing of composite and compomer restoratives: Effectiveness of one-step systems. Oper Dent. 2004;29:275-9

24- Zachrisson BU, Artun J. Enamel surface appearance after various debonding techniques. Am J Orthod. 1979;75:121-37.

25- Zarrinnia K, Eid NM, Kehoe MJ. The effect of different debonding techniques on the enamel surface: an in vitro qualitative study. Am J Orthod Dentofacial Orthop. 1995;108:284-93. 